\title{
Aplicativo Multiplataforma para Planejar Cirurgias de Estrabismo
}

\author{
Igor T. C. Furtado ${ }^{1}$, João D. S. Almeida ${ }^{1}$, Jorge A. Meireles-Teixeira ${ }^{1}$, Geraldo B. Junior ${ }^{1}$ \\ ${ }^{1}$ Núcleo de Computação Aplicada (NCA) - Universidade Federal do Maranhão (UFMA) \\ Caixa Postal 65.085-580 — São Luís — MA — Brasil \\ \{ygortalysson, jorgemeireles1,ge.braz\}@gmail.com, joao.dallyson@ufma.br
}

\begin{abstract}
Strabismus is a pathology that affects about $4 \%$ of the population, causing aesthetic problems and irreversible sensorial changes. Most strabismus cases required surgical treatment for their elimination. Variables involved in the surgical plan are very complex, involving both participation of theoretical scientific knowledge and surgeon experience, which most often uses median values based on formulas and tables. These values do not always apply to all cases. Thus, this work presents a mobile application device for manage and indicate the strabismus surgical plan.
\end{abstract}

Resumo. O estrabismo é uma patologia que afeta cerca de $4 \%$ da população, provocando problemas estéticos e alterações sensoriais irreversíveis. A maioria dos casos de estrabismo exigem tratamento cirúrgico para a sua eliminação. As variáveis envolvidas no plano cirúrgico são complexas, envolvendo tanto a participação do conhecimento científico teórico como a experiência do cirurgião, que na maioria das vezes utiliza valores medianos baseados em fórmulas e tabelas. Estes valores nem sempre se aplicam a todos os casos. Assim, o presente trabalho apresenta um aplicativo para auxiliar os oftalmologistas a gerenciar e planejar cirurgias de estrabismos.

\section{Introdução}

O estrabismo é uma patologia que consiste na ausência do normal paralelismo entre os eixos visuais [Souza-Dias and Almeida 1998]. Esta patologia resulta de uma alteração óculo-motora que impede a fixação bifoveal. A intervenção cirúrgica está indicada para a maioria dos casos com desvios acima de $15 \Delta$ (quinze dioptrias prismáticas). Existem basicamente dois tipos de procedimentos cirúrgicos. No primeiro tipo, podem ser feitos recuos milimétricos da posição original do músculo ocular, ressecção de uma parte dele ou mesmo suturas. Já no segundo, realizam-se ressecções milimétricas do comprimento do músculo ocular, reposicionamento anterior da inserção e pregueamento do tendão muscular. Estes procedimentos são realizados em um ou mais músculos oculares, dependendo do tipo de estrabismo que o paciente apresenta.

É evidente que os avanços da tecnologia móvel melhoram o acesso e auxiliam os profissionais da saúde no diagnóstico e tratamento das patologias. Já existem diversas aplicações oftalmológicas disponíveis e que transformam os smartphones em dispositivos médicos sofisticados [Zvornicanin et al. 2014], porém, essa não é uma realidade dentro da subespecialidade estrabismo, o que dificulta o diagnóstico precoce e o planejamento de cirurgias de estrabismos. 
Diante dessa problemática, apresenta-se um aplicativo móvel multiplataforma para planejar cirurgias de estrabismo. Através de uma interface portátil e de fácil manuseio, o especialista gerencia e elabora seus planos cirúrgicos ou recebe uma sugestão automática de plano com base em técnicas de aprendizado de máquina, utilizando vetores de suporte para regressão (SVR). Para tanto, o aplicativo incorpora a solução proposta por Almeida et al. (2015) para planejar cirurgias de estrabismo através de múltiplos regressores SVR.

O aplicativo oferece, também, a oportunidade de o usuário solicitar a sugestão de plano a outros especialistas, disponibilizando uma terceira opinião, já que o aplicativo fornece a segunda opinião. Por meio dessa colaboração, o aplicativo contribuirá para a melhoria da qualidade do plano cirúrgico elaborado pelos especialistas e, consequentemente, para a prevenção de erros do plano previsto, evitando a necessidade de realização de novo procedimento cirúrgico.

\section{Arquitetura}

A arquitetura utilizada no desenvolvimento do aplicativo está dividida em 3 camadas, como apresentadas na Figura 1. O usuário tem acesso somente à camada de aplicação, onde se encontra o módulo gerenciador do plano cirúrgico, responsável pela manipulação dos planos e organização das informações nas telas da aplicação. A camada de serviços é composta pelos serviços utilizados pelos componentes da camada de aplicação, sendo estes: a ferramenta Qt Creator ${ }^{1}$, o repositório local de arquivos, o módulo de automação e controle do plano cirúrgico e o gerenciador de sincronização. A camada de infraestrutura contém o servidor de banco de dados centralizado e os sistemas operacionais.

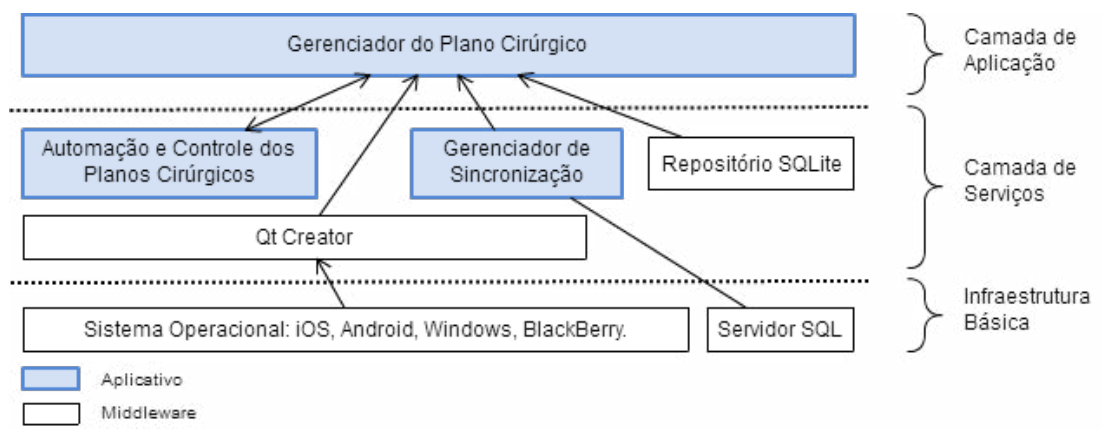

Figura 1. Diagrama da arquitetura da aplicação

Inicialmente, o aplicativo armazena os dados dos planos cirúrgicos gerados em um arquivo local. Em uma versão futura, esses dados serão armazenados e sincronizados em um único repositório central de dados hospedado em nuvem, possibilitando a comunicação e compartilhamento dos planos entre os usuários.

O plano cirúrgico pode ser registrado de duas maneiras: semiautomática, usando a base de planos já realizados; ou manual, baseado na sugestão do próprio especialista. Em ambas, o especialista dependerá dos dados do diagnóstico de cada paciente. Esses dados, provenientes do especialista e do método aqui proposto em Almeida et al. (2015), serão agrupados e submetidos para o algoritmo de aprendizado de máquina supervisionado,

\footnotetext{
${ }^{1}$ http://www.qt.io/
} 
cujos regressores estimarão o valor em milímetros de retrocesso (recuo) ou ressecção que se deve operar em cada músculo ocular.

\section{Desenvolvimento do Aplicativo}

Na tela inicial da aplicação, foi definido um botão para controlar o acesso do especialista ao aplicativo (Figura-2a), sendo que, de acordo com os requisitos, o usuário só poderá criar e gerenciar os planos cirúrgicos se estiver devidamente cadastrado (Figura-2b). O cadastro é composto por dados básicos do especialista (Figura-2c), somente para controle das informações. Qualquer plano gerado no programa estará relacionado ao respectivo usuário.

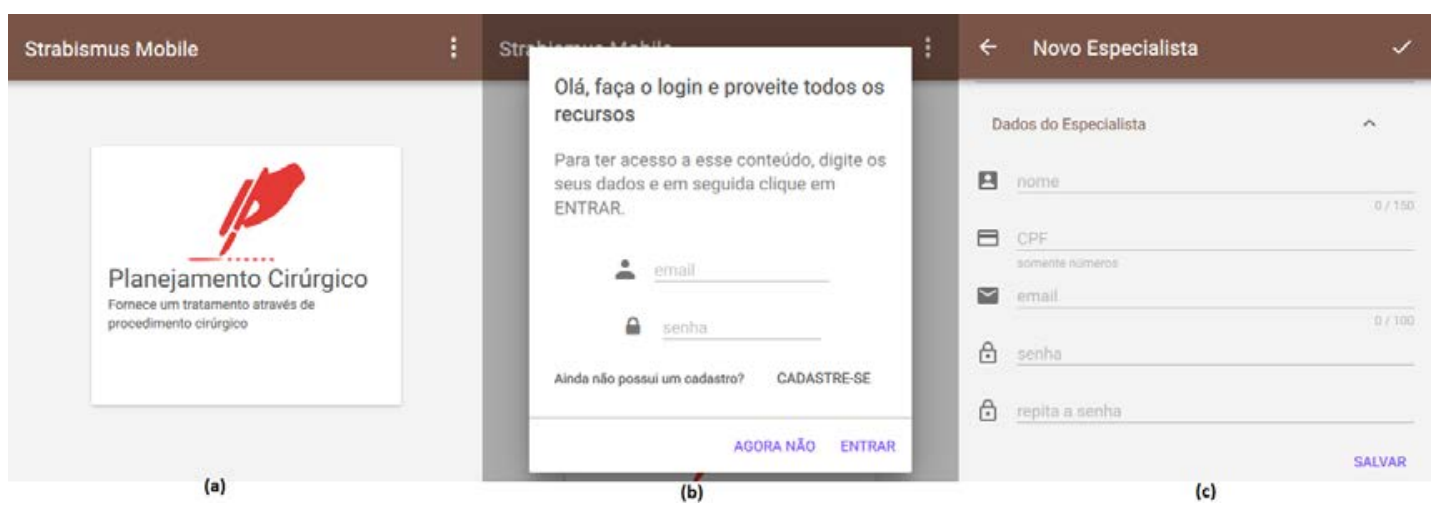

Figura 2. Telas iniciais da aplicação: (a)Tela inicial, (b)Acesso à aplicação, (c)Cadastro do especialista.

Após realizar o acesso, o especialista tem a opção de criar um novo plano cirúrgico ou manipular os já armazenados na base local, mesmo para aqueles gerados automaticamente pelo método computacional. Caso queira criar o seu próprio plano, precisará dispor dos dados do paciente diagnosticado com estrabismo (Figura-3) e, em seguida, preencher obrigatoriamente todos os campos antes de clicar em salvar.

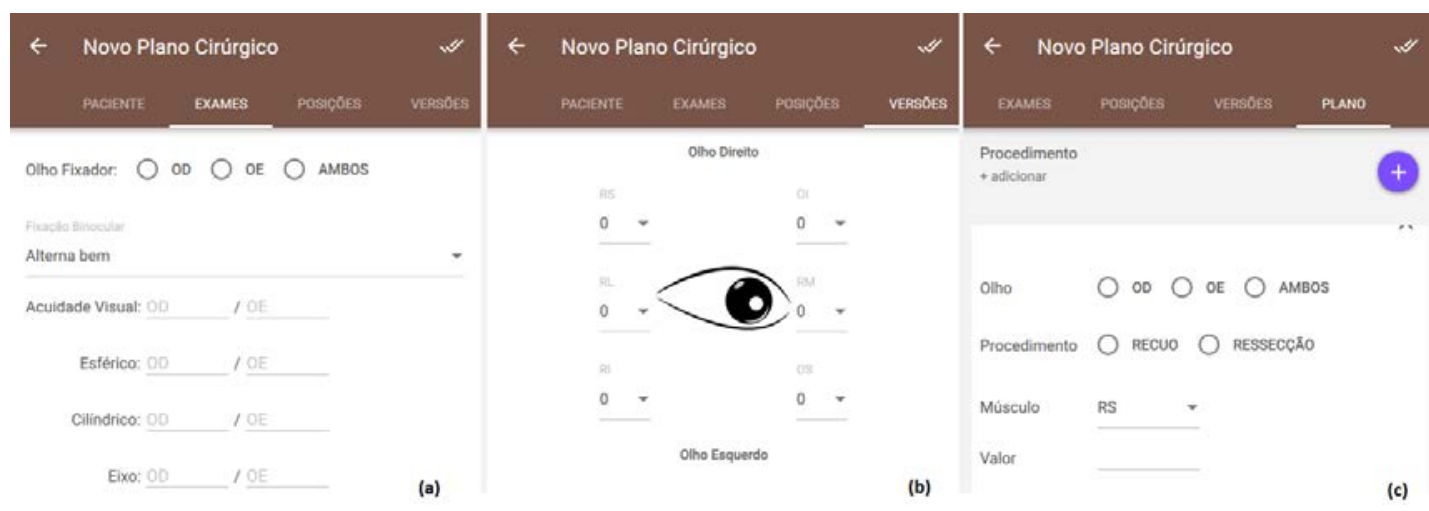

Figura 3. Telas de criação de um novo plano cirúrgico elaborado pelo especialista: (a)Dados do diagnóstico do paciente, (b) Dados das versões, (c) Dados do procedimento a ser aplicado.

Um mesmo paciente pode ter tantos planos cirúrgicos quantos forem necessários para que o especialista tenha certeza de qual método cirúrgico aplicar. Em seu auxílio, 
poderá criar um plano automaticamente, usando o mesmo diagnóstico para comparar se o seu procedimento é parecido com o sugerido pelo aplicativo.

A geração automática dos planos, ainda em desenvolvimento, utiliza técnicas de aprendizado de máquina supervisionada. Por conta disso, fez-se necessário obter uma base de dados de prontuários de pacientes que já foram submetidos a procedimentos cirúrgicos. De cada paciente, foram extraídas 23 características: fixação binocular, equivalente esférico direito e esquerdo, desvios das cinco posições diagnósticas, tipo de desvio, acuidade visual e as 12 medidas de versões dos olhos esquerdo e direito. Estas características foram escolhidos conforme relevância da informação para o plano cirúrgico elaborado pelo especialista [Almeida et al. 2015].

Após passar por um processo de normalização para uma faixa de valores comuns entre -1 e 1, estima-se o plano cirúrgico para cada diagnóstico de paciente utilizando os regressores SVR treinados com os mesmos parâmetros utilizados por Almeida et al. (2015). A estimação do plano é realizada de forma instantânea.

\section{Considerações Finais}

Este trabalho apresentou uma ferramenta para gerenciar planos de cirurgia de estrabismo, servindo tanto para auxiliar o especialista como para treinar profissionais da oftalmologia e médicos residentes, uma vez que estes últimos poderão utilizar os planos indicados pelo aplicativo ou o plano sugerido em colaboração por outro especialista como um tira-provas.

Certamente, apesar de estar ainda em desenvolvimento, podem-se antever diversas melhorias em versões futuras do aplicativo. Por exemplo, a integração com banco de dados em nuvem e, consequentemente, a atualização das informações em tempo real, permitindo, assim, a comunicação entre os especialistas e demais usuários do sistema. Citem-se ainda a inclusão e a integração com módulo de diagnóstico automático do estrabismo em imagens para fornecer as medidas do desvio, tipo de estrabismo e olho fixador.

Por fim, vale destacar que após a realização dos devidos testes qualitativos e de validação do aplicativo, este poderá ser utilizado nos mais diversos centros oftalmológicos. A utilização do aplicativo nestes centros poderá contribuir para uma melhor gestão dos recursos financeiros. Faz-se a ressalva de que o objetivo não é substituir o especialista, mas oferecer-lhe suporte por meio de outras opiniões na elaboração do plano cirúrgico.

\section{Agradecimentos}

Os autores agradecem à FAPEMA e ao CNPq, pelo apoio financeiro.

\section{Referências}

Almeida, J. D. S., Silva, A. C., Teixeira, J. A. M., Paiva, A. C., and Gattass, M. (2015). Surgical planning for horizontal strabismus using support vector regression. Computers in biology and medicine, 63:178-186.

Souza-Dias, C. and Almeida, H. (1998). Estrabismo. Conselho Brasileiro de Oftalmologia. São Paulo: Rocca, pages 12-128.

Zvornicanin, E., Zvornicanin, J., and Hadziefendic, B. (2014). The use of smart phones in ophthalmology. Acta Informatica Medica, 22(3):206. 